\title{
Analgesic Effect of Intrathecal Gabapentin in a Rat Model of Persistent Muscle Pain
}

\author{
Tae-Wook Kang, MD ${ }^{1}$, Min Kyun Sohn, MD $^{2}$, Noh Kyoung Park, MD ${ }^{1}$, Sang Hyung Ko, MD $^{1}$, \\ Kyoung Jin Cho, $\mathrm{MD}^{1}$, Jaewon Beom, $\mathrm{MD}^{2}$, Sangkuk Kang, $\mathrm{MD}^{1}$ \\ ${ }^{1}$ Department of Physical Medicine and Rehabilitation, Sun General Hospital, Daejeon; \\ ${ }^{2}$ Department of Physical Medicine and Rehabilitation, Chungnam National University Hospital, Daejeon, Korea
}

\begin{abstract}
Objective To evaluate the analgesic effect of intrathecal gabapentin therapy on secondary hyperalgesia in a rat model of persistent muscle pain.

Methods Intrathecal catheters were implanted into rats. Mechanical secondary hyperalgesia was induced by repeated intramuscular injections of acidic solution into the gastrocnemius muscle. Gabapentin was administrated intrathecally. Rats were allocated to control and experimental (gabapentin 30, 100, 300, and 1,000 $\mu \mathrm{g}$ group. After gabapentin administration, mechanical withdrawal threshold was measured every 15 minutes and the motor function was measured 30 minutes later.

Results Mechanical hyperalgesia was evoked after the second acidic buffer injection. There was a significant improvement on the mechanical threshold after administration of 100, 300, and 1,000 $\mu$ g gabapentin compared to pre-injection and the control group. The analgesic effect continued for 105, 135, and 210 minutes, respectively. To discern side effects, motor function was measured. Motor function was preserved in both groups after gabapentin administration, except for rats who received $1,000 \mu \mathrm{g}$ gabapentin.

Conclusion Intrathecal gabapentin administration produces dose-dependent improvements in mechanical hyperalgesia in a persistent muscle pain rat model. This implicates the central nervous system as having a strong influence on the development of persistent mechanical hyperalgesia. These results are helpful in understanding the pathophysiology of secondary hyperalgesia and in the treatment of patients with chronic muscle pain.
\end{abstract}

Keywords Analgesic effect, Intrathecal gabapentin injection, Persistent muscle pain

Received March 12, 2014; Accepted July 16, 2014

Corresponding author: Sangkuk Kang

Department of Physical Medicine and Rehabilitation, Sun General Hospital, 29, Mokjung-ro, Jung-gu, Daejeon 301-725, Korea

Tel: +82-42-220-8468, Fax: +82-303-3130-8468, E-mail: rmactksk@daum. net

(c) This is an open-access article distributed under the terms of the Creative Commons Attribution Non-Commercial License (http://creativecommons. org/licenses/by-nc/3.0) which permits unrestricted noncommercial use, distribution, and reproduction in any medium, provided the original work is properly cited.

Copyright $\odot 2014$ by Korean Academy of Rehabilitation Medicine

\section{INTRODUCTION}

Muscles account for $40 \%$ of body volume and are the most common site of pain. Virtually everyone has or will experience muscle pain. Musculoskeletal pain, such as myofascial pain syndrome and fibromyalgia, is clinically quite common. But, the pain can become chronic due to the lack of awareness of treatment and pain evaluation. Acute muscle pains caused by a musculoskeletal injury producing an afferent nociceptive stimulus commonly respond well to treatment. But, if allowed to become 
chronic, unpleasant emotional responses are likely to develop beyond the pathological damage of the pain itself [1]. Objective research on patients with chronic pain is difficult because of the variety of parameters like emotional state, sleep, and medication dosages [2].

The intramuscular injection of acidic solution in experimental pain models have been developed as a method for chronic pain studies. Sluka et al. [3] induced a mechanical hyperalgesia lasting 3-4 weeks in rats by two intramuscular injections of acidic buffer in the gastrocnemius muscles. Similarly, Sohn et al. [2] produced a persistent pain rat model with the bilateral mechanical hyperalgesia lasting for about 4 weeks by two intramuscular injections of acidic buffer in the gastrocnemius muscles. These observations support the view that the central nervous system is responsible for the formation of the chronic pain. A follow-up study suggested that increased activities of wide dynamic range cells and high threshold cells of the dorsal horn cause persistent muscle pain $[4,5]$. Presently, we specifically addressed this issue by assessing spinal level with intrathecal gabapentin administration.

The medications for the neuropathic pain caused by a nervous sensitization primarily include non-steroidal analgesic drugs. Secondary drugs include antidepressants and anticonvulsants, while tertiary drugs include baclofen, clonidine, capsaicin, and morphine [6]. Gabapentin is an effective anticonvulsant drug that is structurally related to $\gamma$-aminobutyric acid. The main targets of gabapentin binding are the $\alpha 2 \delta$-1 subunits of the voltagegated calcium channel located on dorsal root ganglion neurons. This binding subsequently reduces neurotransmitter release and alleviates neuronal excitation $[7,8]$. Gabapentin is used to treat neuropathic pain, such as postherpetic neuralgia [9], neuropathic pain following spinal cord injuries [10], phantom limb pain [11], and diabetic polyneuropathy [12].

This study is designed to evaluate the analgesic effects of the intrathecal gabapentin therapy on secondary hyperalgesia in a persistent pain rat model.

\section{MATERIALS AND METHODS}

\section{Intrathecal catheter insertion}

Male Sprague-Dawley rats, 8 weeks of age, weighing 250-300 g were the experimental animals. They were

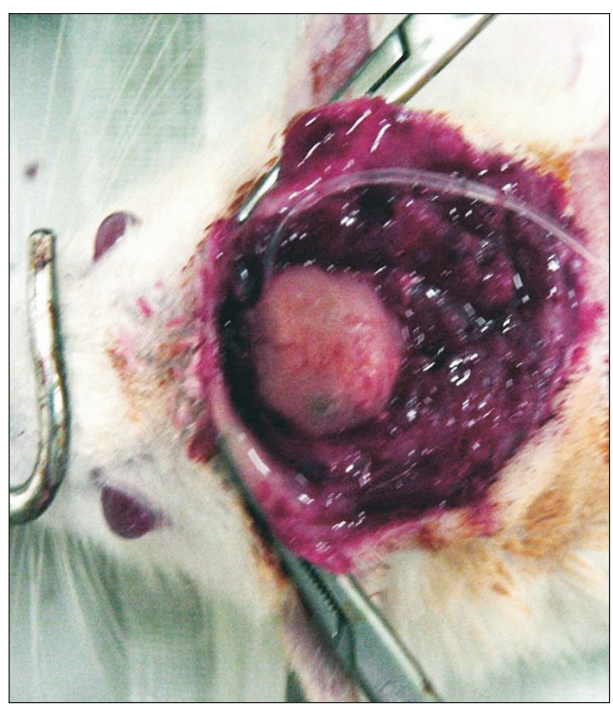

Fig. 1. Intrathecal catheter insertion for the administration of drugs into the subarachnoid space.

housed at a temperature of $23.0^{\circ} \mathrm{C} \pm 1.0^{\circ} \mathrm{C}$ and humidity of $50 \% \pm 3 \%$ with alternating 12 -hour light-dark cycle and had free access to food and water. A mixture of ketamine and xylazine was injected into the abdominal cavity of each rat as anesthesia, while the head was fixed in position using a stereotactic head holder. For the catheterization, the occiput and posterior neck region were shaved and sterilized with povidone-iodine. A dural incision was made at the atlantooccipital membrane, into which a polyethylene catheter was inserted $8-8.5 \mathrm{~cm}$ to lumbar enlargement as described previously [13]. The tip of the catheter was plugged with a 28-gauge short steel wire and was fixed at the upper skull (Fig. 1). The incision area was sutured with silk before awakening the rats.

\section{Modeling persistent muscle pain}

Rats with the inserted plugged catheter were observed for 4 days of their recovery. Any rat showing motor disturbance after 4 days was excluded. All remaining rats were anesthetized by inhaling halothane. Each left hind limb was shaved and sterilized with povidone-iodine. Intramuscular injection of $250 \mu \mathrm{L}$ of $\mathrm{pH} 6.0$ phosphate buffered saline was done in the left gastrocnemius muscle. Secondary injection was conducted with the same solution 3 days after the first injection. Behavioral examination was carried out (see below) 4 hours after the second injection. After examination, the mechanical thresholds of select rats were $32-40 \mathrm{mN}$. 


\section{Behavioral examination}

Behavioral examinations in assessing the mechanical thresholds were conducted through the von Frey filaments. For evaluation, rats were transferred to steel cages and were allowed to acclimate for 10 minutes. Mechanical withdrawal thresholds were assessed through the responses of rats with a series of filaments with the bending forces of 8, 32, 60, 75, 120, 187, and $358 \mathrm{mN}$. The filaments were applied vertically to the left hind paws for 4 seconds while the filament was bent. Brisk withdrawal or paw flinching was considered to be a positive response. In the absence of a response, the filament of next greater force was applied. The mechanical stimulus producing a $50 \%$ likelihood of withdrawal response in 10 tests was determined using the up-and-down method [14].

\section{Administration of gabapentin}

Twenty-four hours after the second injection of acidic buffer, rats were divided into the control and experimental groups. Within the experimental group, subgroups were formed on the basis of gabapentin dose $(30,100$, 300 , and 1,000 $\mu \mathrm{g}$, respectively). Each dose of gabapentin (Sigma-Aldrich, St. Louis, MO, USA) was dissolved in 10 $\mu \mathrm{L}$ of normal saline and was injected through the catheter in each experimental group. The control group was administered $20 \mu \mathrm{L}$ of saline. Each group was composed of eight rats. The behavioral examination was conducted

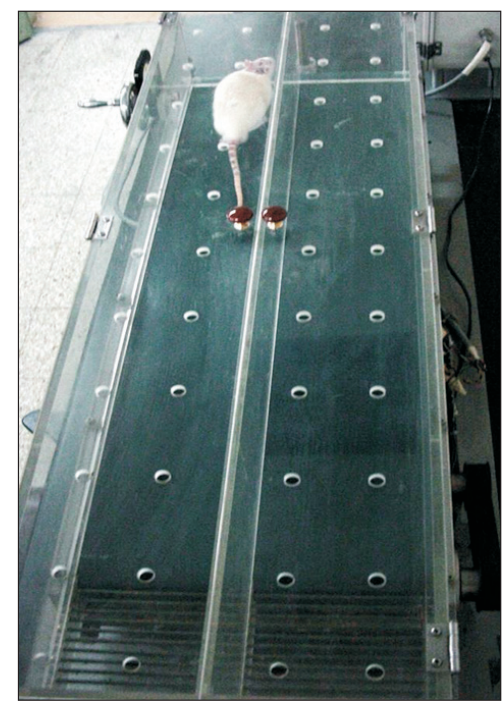

Fig. 2. Treadmill motor function test determined by measurements on exercise time at $5^{\circ}$ inclination and $17 \mathrm{~m} /$ min speed. before and every 15 minutes for 4 hours after the intrathecal injection.

\section{Motor function test}

Each group was tested for the motor function with the treadmill (Daejong E\&C, Daejeon, Korea) before and 30 minutes after receiving gabapentin. The treadmill was equipped with electrified steel bars on the lower part to make rats continue running forward (Fig. 2). As a preliminary exercise for adaptation to the treadmill, all rats were subjected to a daily 5 -minute exercise at $5^{\circ}$ inclination and a treadmill speed of $15 \mathrm{~m} / \mathrm{min}$ for 2 days prior to the first gabapentin injection. In the motor function tests, the treadmill was slowly sped up to $17 \mathrm{~m} / \mathrm{min}$ at a $5^{\circ}$ inclination, which was then maintained. The run times were measured, and the test was ended if a rat ran more than 150 seconds.

\section{Statistical analyses}

Non-parametric testing was done using SPSS ver. 18.0 for Windows (SPSS Inc., Chicago, IL, USA). Wilcoxon signed-rank test was used to determine the statistical significance of the mechanical threshold and motor function. After gabapentin administration, Friedman test was conducted within groups and Kruskal-Wallis test was conducted between groups. If there was a statistical significance, the Dunnett test was conducted for multiple comparisons.

\section{RESULTS}

\section{Mechanical hyperalgesia}

All subjects were measured of their mechanical thresholds with von Frey filaments. The mean threshold at the limb was $336.63 \pm 9.06 \mathrm{mN}$ before acidic buffer injection and decreased to $39.70 \pm 2.00 \mathrm{mN}$ by 4 hours after the second buffer injection $(\mathrm{p}<0.05)$. Mechanical hyperalgesia was induced in ipsilateral limbs.

\section{Effect of gabapentin on mechanical stimulus}

Significant changes were evident in the thresholds after administration of 100,300 , and 1,000 $\mu$ g gabapentin. Each group showed improvements in the mechanical threshold after 15 minutes. The improvements lasted for 105,135 , and 210 minutes, respectively $(\mathrm{p}<0.05)$. No statistical significance was apparent using $30 \mu \mathrm{g}$ gabapentin. 


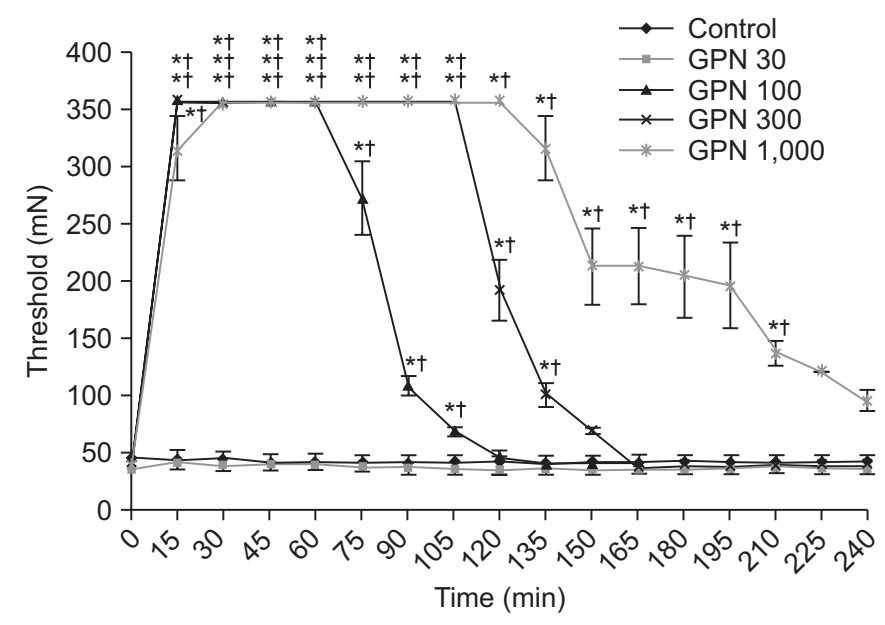

Fig. 3. Withdrawal thresholds of the hind paw to the von Frey filaments with respect to give to the amount of intrathecal gabapentin (GPN) injections. ${ }^{*} p<0.05$, compared with that before the injection. ${ }^{\dagger} p<0.05$, compared with the controlled group.

Compared with the control group, rats receiving 100, 300, and $1,000 \mu \mathrm{g}$ gabapentin showed significant changes in the threshold (Fig. 3).

\section{Motor function test}

The motor function showed no significant difference following injection of 30,100 , and $300 \mu \mathrm{g}$ gabapentin. In rats who received $1.000 \mu \mathrm{g}$ gabapentin the run time of the subjects on the treadmill was significantly decreased $(\mathrm{p}<0.05)$ compared with the prior preliminary exercise test and the control group (Fig. 4).

\section{DISCUSSION}

Several methods employing agents including hypertonic saline and hyaluronic acid have been developed to derive experimental models on musculoskeletal pain. These models showed that pain can be induced immediately following injections and persisting for 15 to 20 minutes $[15,16]$. In one study [3], the authors claimed that two injections of $\mathrm{pH} 4.0$ acidic saline given 2 and 5 days apart produced a significant mechanical hyperalgesia that lasted 4 weeks. Hyperalgesia was not maintained with injections given 10 days apart. Similarly, Sohn et al. [2] developed a persistent pain rat model with mechanical hyperalgesia in the bilateral hind limbs that could persist about 4 weeks through two injections of $\mathrm{pH} 6.0$ acidic buffer given in 3 days apart. This model did not

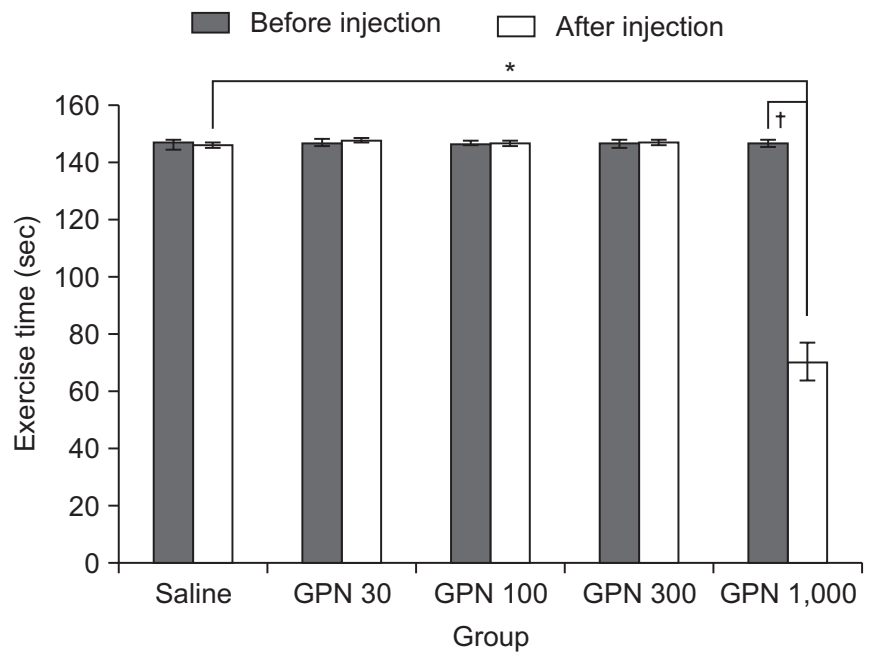

Fig. 4. Treadmill exercise time before and after the intrathecal gabapentin (GPN) injection. * $\mathrm{p}<0.05$, between the experimental and the controlled groups. ${ }^{\dagger} \mathrm{p}<0.05$, between before and after the intrathecal gabapentin injection.

produce an electrophysiologic dysfunction. In this study, mechanical hyperalgesia was provoked through injections of acidic buffer [2].

Gabapentin is generally used to treat neuropathic pains in both the central and the peripheral nervous systems [8]. In the peripheral nerve system, the pain inhibitory mechanism consists of several steps. Gabapentin is anchored with $\alpha 2 \delta$-1 subunits of voltage-gated calcium channel in the dorsal root ganglion, and effectively blocks the influx of $\mathrm{Ca}^{2+}$. This binding reduces the secretion of glutamate, aspartate, and $\mathrm{P}$ substance, which leads to the inhibition of $\alpha$-amino-3-hydroxy-5-methyl4-isoxazolepropionic acid and $\mathrm{N}$-methyl-D-aspartate receptors, and disrupts nuclear factor kappa-light-chainenhancer of activated B cells (NF- $\kappa \mathrm{B}$ ), which is involved in the generation of cytokines [7,8,17-19]. In the central nervous system, gabapentin reduces the presynaptic secretion of gamma-amino butyric acid, while increasing the secretion of glutamate and noradrenaline. This mechanism alleviates pain by stimulating the descending inhibitions [20].

Central sensitization represents an enhanced function of neurons and circuits in nociceptive pathways caused by increased membrane excitability, synaptic efficacy, and reduced inhibition. It demonstrates the remarkable plasticity of the somatosensory nervous system in response to activity, inflammation, and neural injury [5,21]. 
Microglial cells are involved in the formation of central sensitizations. The cells release mediators that modulate spinal cord synaptic transmissions and increase the excitability of dorsal horn neurons [22]. Also, gabapentin reverses microglial cell activation, and alleviates hyperalgesia $[23,24]$.

Intrathecal gabapentin administration was used in several animal models to relieve neuropathic pains. Rat models of thermal injury under the inhibition of hyperalgesia, the spinal nerve ligation under the reduction of mechanical and thermal hyperalgesia, and the nerve ligation-induced pain with the prevention of hyperalgesia effects have been reported prior to our study [25-27]. In these models, intrathecal gabapentin given at doses of 100 and $300 \mu \mathrm{g}$ alleviated hyperalgesia for about 60 minutes $[25,26]$. Our study demonstrated significant improvements of the mechanical threshold in rats receiving 100,300 , and 1,000 $\mu \mathrm{g}$ compared with before administration and the control group. The analgesic effect at the respective doses continued for 105, 135, and 210 minutes.

Suppression of hyperalgesia was also observed in studies involving the systematic injection of gabapentin. Intravenous injection of $90 \mathrm{mg} / \mathrm{kg}$ gabapentin reportedly alleviated mechanical hyperalgesia for 120 minutes [28]. The relief could be extended to up to 140 minutes using an intraperitoneal injection of $100 \mathrm{mg} / \mathrm{kg}$ of gabapentin [29]. In our study, the suppression of hyperalgesia could have been preserved much longer than prior studies with smaller injection doses.

Intravenous injection of various doses of gabapentin reportedly elicited a dose-dependent attenuation of allodynia in nerve-injured rats, while a high dose $(90 \mathrm{mg} / \mathrm{kg})$ had a sedative effect [28]. In our study, 1,000 $\mu$ g gabapentin decreased motor function at a dose of $3.3 \mathrm{mg} / \mathrm{kg}$. This implies that intrathecal administration has more potential of complications than intravenous administration. Rats given $1,000 \mu$ g gabapentin displayed a shorter treadmill exercise time, but exhibited the same withdrawal response from the $358 \mathrm{mN}$ von Frey filament as rats receiving 100 and $300 \mu \mathrm{g}$ gabapentin. Higher doses up to $1,000 \mu \mathrm{g}$ did not increase the pain threshold but did worsen the motor function, which is a hallmark of the ceiling effect [30].

Ipsilateral painful stimulus leads to the mechanical hyperalgesia of bilateral limbs in the persistent muscle pain model. Wide dynamic range neurons and high threshold neurons of dorsal horn cells in the spinal cord are sensi- tized in the persistent muscle pain model $[4,5]$. The afferent nociceptive stimulus flows in the dorsal horn cells and passes through the spinothalamic tract to the supraspinal level. Hence, intrathecal injections were adopted to treat persistent muscle pain. But, the supraspinal level can mediate the sensitization. Further studies are required to investigate the changes of them.

In our study, intrathecal gabapentin was employed as a neuropathic pain treatment in persistent muscle pain and to alleviate hyperalgesia. Symptoms of chronic muscle pain, such as hyperalgesia, formed through simple muscle injury and nervous sensitization. Neuropathic pain medications could have an important role in the treatment of patients with persistent muscle pain.

Limitations of our study were the small number of subjects due to the large number of subgroups with varying doses, and the large dose interval between 300 and 1,000 $\mu$ g. Further study with more subjects and smaller dose intervals for assessing the reasonable therapeutic range without complications is required. Another limitation is that the study was conducted quite promptly before the definitive confirmation on the progress of muscle pain on the subjects. The progress of muscle pain had been confirmed in prior studies of a persistent muscle pain rat model, and thus the duration of muscle pain was not monitored in this study. Further research with the confirmation of the pain progress could be considered more suitable for the study on chronic muscle pain.

In conclusion, intrathecal gabapentin administration showed a dose-dependent improvement of mechanical hyperalgesia of ipsilateral limbs in the persistent muscle pain rat model. This means that the central nervous system is responsible for the formation of the persistent mechanical hyperalgesia. These results are helpful in understanding of the pathophysiology of secondary hyperalgesia and in the treatment of patients with chronic muscle pain.

\section{CONFLICT OF INTERST}

No potential conflict of interest relevant to this article was reported.

\section{REFERENCES}

1. Kim C. Chronic pain syndrome. J Korean Assoc Pain 
Med 2003;2:17-24.

2. Sohn MK, Lee KY, Yang SS, Lee SH, Kim SS. Development and assessment of animal model of persistent muscle pain. J Korean Acad Rehabil Med 2003;27:75763.

3. Sluka KA, Kalra A, Moore SA. Unilateral intramuscular injections of acidic saline produce a bilateral, longlasting hyperalgesia. Muscle Nerve 2001;24:37-46.

4. Sohn MK, Kang SK, Lee SH, Han SM. The mechanism of contralateral hyperalgesia in the persistent muscle pain rat model. J Korean Acad Rehabil Med 2004;28: 477-82.

5. Sohn MK, Yoon YS, Lee SH, Kang SK, Kim BO, Sohn $\mathrm{JH}$. The effect of low-level laser irradiation on activities of dorsal horn neurons in rats with experimental muscle pain. J Korean Acad Rehabil Med 2005;29:51320.

6. Suh DW, Kim SJ. Effects of drugs for pain relief on neuropathic animal model. J Korean Acad Rehabil Med 1996;20:17-27.

7. Fink K, Meder W, Dooley DJ, Gothert M. Inhibition of neuronal $\mathrm{Ca}(2+)$ influx by gabapentin and subsequent reduction of neurotransmitter release from rat neocortical slices. Br J Pharmacol 2000;130:900-6.

8. Kukkar A, Bali A, Singh N, Jaggi AS. Implications and mechanism of action of gabapentin in neuropathic pain. Arch Pharm Res 2013;36:237-51.

9. Segal AZ, Rordorf G. Gabapentin as a novel treatment for postherpetic neuralgia. Neurology 1996;46:1175-6

10. Levendoglu F, Ogun CO, Ozerbil O, Ogun TC, Ugurlu $\mathrm{H}$. Gabapentin is a first line drug for the treatment of neuropathic pain in spinal cord injury. Spine (Phila Pa 1976) 2004;29:743-51.

11. Bone M, Critchley P, Buggy DJ. Gabapentin in postamputation phantom limb pain: a randomized, double-blind, placebo-controlled, cross-over study. Reg Anesth Pain Med 2002;27:481-6.

12. Backonja M, Beydoun A, Edwards KR, Schwartz SL, Fonseca V, Hes M, et al. Gabapentin for the symptomatic treatment of painful neuropathy in patients with diabetes mellitus: a randomized controlled trial. JAMA 1998;280:1831-6.

13. Yaksh TL, Rudy TA. Chronic catheterization of the spinal subarachnoid space. Physiol Behav 1976;17:10316.

14. Chaplan SR, Bach FW, Pogrel JW, Chung JM, Yaksh
TL. Quantitative assessment of tactile allodynia in the rat paw. J Neurosci Methods 1994;53:55-63.

15. Graven-Nielsen T, Arendt-Nielsen L, Svensson P, Jensen TS. Quantification of local and referred muscle pain in humans after sequential i.m. injections of hypertonic saline. Pain 1997;69:111-7.

16. Hamamoto DT, Ortiz-Gonzalez XR, Honda JM, Kajander KC. Intraplantar injection of hyaluronic acid at low $\mathrm{pH}$ into the rat hindpaw produces tissue acidosis and enhances withdrawal responses to mechanical stimuli. Pain 1998;74:225-34.

17. Kim YS, Chang HK, Lee JW, Sung YH, Kim SE, Shin MS, et al. Protective effect of gabapentin on N-methyl$\mathrm{D}$-aspartate-induced excitotoxicity in rat hippocampal CAl neurons. J Pharmacol Sci 2009;109:144-7.

18. Park S, Ahn ES, Han DW, Lee JH, Min KT, Kim H, et al. Pregabalin and gabapentin inhibit substance Pinduced NF-kappaB activation in neuroblastoma and glioma cells. J Cell Biochem 2008;105:414-23.

19. Bockbrader HN, Wesche D, Miller R, Chapel S, Janiczek N, Burger P. A comparison of the pharmacokinetics and pharmacodynamics of pregabalin and gabapentin. Clin Pharmacokinet 2010;49:661-9.

20. Takasu K, Ono H, Tanabe M. Gabapentin produces PKA-dependent pre-synaptic inhibition of GABAergic synaptic transmission in LC neurons following partial nerve injury in mice. J Neurochem 2008;105:933-42.

21. Latremoliere A, Woolf CJ. Central sensitization: a generator of pain hypersensitivity by central neural plasticity. J Pain 2009;10:895-926.

22. Wen YR, Tan PH, Cheng JK, Liu YC, Ji RR. Microglia: a promising target for treating neuropathic and postoperative pain, and morphine tolerance. J Formos Med Assoc 2011;110:487-94.

23. Wodarski R, Clark AK, Grist J, Marchand F, Malcangio M. Gabapentin reverses microglial activation in the spinal cord of streptozotocin-induced diabetic rats. Eur J Pain 2009;13:807-11.

24. Yang JL, Xu B, Li SS, Zhang WS, Xu H, Deng XM, et al. Gabapentin reduces CX3CL1 signaling and blocks spinal microglial activation in monoarthritic rats. Mol Brain 2012;5:18.

25. Jun JH, Yaksh TL. The effect of intrathecal gabapentin and 3-isobutyl gamma-aminobutyric acid on the hyperalgesia observed after thermal injury in the rat. Anesth Analg 1998;86:348-54. 
26. Cho HS, Kim MH, Choi DH, Lee JI, Gwak MS, Hahm TS. The effect of intrathecal gabapentin on mechanical and thermal hyperalgesia in neuropathic rats induced by spinal nerve ligation. J Korean Med Sci 2002;17:225-9.

27. Chu LC, Tsaur ML, Lin CS, Hung YC, Wang TY, Chen $\mathrm{CC}$, et al. Chronic intrathecal infusion of gabapentin prevents nerve ligation-induced pain in rats. Br J Anaesth 2011;106:699-705.

28. Pan HL, Eisenach JC, Chen SR. Gabapentin suppresses ectopic nerve discharges and reverses allodynia in neuropathic rats. J Pharmacol Exp Ther 1999;288:
1026-30.

29. Kayser V, Christensen D. Antinociceptive effect of systemic gabapentin in mononeuropathic rats, depends on stimulus characteristics and level of test integration. Pain 2000;88:53-60.

30. Pandey CK, Navkar DV, Giri PJ, Raza M, Behari S, Singh RB, et al. Evaluation of the optimal preemptive dose of gabapentin for postoperative pain relief after lumbar diskectomy: a randomized, double-blind, placebo-controlled study. J Neurosurg Anesthesiol 2005; 17:65-8. 